\title{
Editorial
}

\section{Exactly 250 Feet}

\author{
James T. Lee, MD, PhD
}

\section{Accuracy means something to me.-Charles A. Lindbergh}

In this issue of Infection Control and Hospital Epidemiology, Zanetti et al. describe a simple clinical trial in the field of cardiac surgery process improvement. ${ }^{1}$ Underpinning their study is the tenet that, in certain circumstances, failure to redose intraoperatively a preoperatively infused prophylactic antimicrobial agent is a process step error. This kind of error should be absolutely preventable. Under actual operating room conditions, these investigators probed the value of an intraoperative alerting function that signaled a circulating nurse, via computer, a few minutes before the second dose of prophylactic agent was indicated. On-schedule redosing occurred more often during cases randomized to use of the alerting function than during control cases in which no alert was given. This thought-provoking article should be digested by everyone interested in surgical-site infections.

I first saw this unusual report in manuscript form while strapped into an airliner seat, flying through bad weather at night over Alaska. Analogical thinking ensued. How does a flight crew safely land its plane if weather at the destination is so foul that the runway can't be seen until just before touchdown?

Two issues lurk here. One issue is getting the plane onto the appropriate magnetic heading that leads to the runway with the plane lined up on that runway's centerline. The second issue is compound-timing the beginning of descent, then maintaining a steady descent rate all the way down (pilots call this a stabilized approach) so that the plane is gently sinking just as it comes over the proximal end of the runway at an air speed not too far above its stalling speed. When ceiling and visibility are "at minimums," safe runway contact requires a special interplay of technology, human behavior, and immutable laws of physics. Day or night, heightened gravitas surrounds instrument landing operations in bad weather.
Instrument landing system approaches, like surgical operations, are serious activities that entail a flow of coordinated steps. In the United States, the instrument landing system decision height is usually 200 feet. Just above decision height, things are somber in the cockpit because the airliner is moving at 120 to 130 miles per hour, settling toward terra firma at 10 to 12 feet per second. If the runway, its markings, and/or certain lights are not seen at decision height and if aircraft attitude is not conducive to landing, a go-around and missed approach procedure must start immediately. ${ }^{2}$

Enter the radar altimeter, an accurate instrument in the plane that continuously displays digitally the height of the main landing gear above terrain. Radar altimetry is not always required for identifying decision height, but it can be an important back-up gadget. ${ }^{3}$ Here comes the punch line: Before the approach begins, a radar altimeter can be set by the aircrew to automatically sound audible alerts in the cockpit. For illustration, an alert sounding at 250 feet reminds the crew that arrival at the 200-foot decision height is coming in a few seconds, facilitating smooth, nonpanicked reactions. Readers who wonder what happens in modern "glass cockpits" should pause to visit an interesting web site. ${ }^{4}$ Its content is not recommended for anxious Airbus riders.

The Zanetti team's redosing alert gadgetry (optionally preset by personnel, running in the background, alerting accurately, and supporting an important decision) mimics the use of radar altimetry during an instrument landing system approach. Aviation and surgical care have in common the necessity to do things exactly right at exactly the right time by optimally using various human-machine and human-human interfaces. Near-zero outcome flaw rates are expected in each profession. Logically, near-zero process step errors are pursued by both. Automated reminder gadgets are appropriate amenities in both cockpits and operating rooms, but surgery has not done a crisp 
job of importing many good ideas from aviation. It's not easy to craft and articulate useful analogical arguments. I have heard high-profile speakers, including NASA astronauts, do a rather weak job in that regard. Another piece of the problem is that nurses, anesthetists, and surgeons may not know enough aviation esoterica for even perfect analogical points to sink in.

The article's opening salvo says that, "Perioperative antibiotic prophylaxis is among the most effective methods to reduce the incidence of surgical-site infections for many types of surgical procedures." The authors should have used the word "some" in place of "many" and might have substituted the word "misunderstood" for the word "effective."

Cynics will assert that a cheap kitchen timer, digital wristwatch, or cuckoo clock could just as well have been used by the circulating nurse to sense the passage of 225 minutes. However, we can appreciate that the "irritation function" (freezing of the computer screen after an alert) motivated the circulator to pester anesthesia folks to see whether a redosing plan was afoot, then to repester them later to ensure infusion of the redose. Are the authors asking us to make a simple task way too complicated by bringing a computer and this two-person, two-conversation, tagteam, to-and-fro, asymmetrical hectoring into the fracas? Certified nurse anesthetists and anesthesiologists everywhere are accustomed to giving all sorts of intravenous drugs throughout an operation and keeping neat records of the drug names, doses, and timing as things roll along. A yellow highlighter streak marked preoperatively at the appropriate time line on the anesthesia record sheet can also serve as a reminder to give a cefazolin redose. I wonder how many readers have actually visited active cardiac operating rooms to "shadow" (New Age term) the anesthesia "providers" (another New Age term). At our little institution, ${ }^{5}$ operating room "culture" (yikes, a third New Age term) is such that anesthesia nurses peek over the drapes to lobby surgeons for repeat prophylaxis doses, and that's not just during cardiac work. In the cockpit of a modern airliner about to descend-even in perfectly clear weatherthere is a formal briefing of the approach: Who will do what and when. This is carried out, literally, by the book (a checklist is used), even though crews can recite its steps from memory. It's time for this aspect of cockpit culture to be brought to the surgery-anesthesia interface.

The term "surgical-site infection" is used several times, but we never learn the numbers of mediastinitis diagnoses, superficial incisional infections, or deep incisional infections. Two-thirds of the operations were not coronary artery bypass procedures, which eliminated 177 potential garden-variety saphenous vein harvest incision infections. I presume that 273 study patients had sternotomy incisions. Was the authors' hospital in the midst of some horrible mediastinitis problem in the months preceding the study?

The article is mum regarding cefazolin dose sizes, either preoperatively or intraoperatively, or whether any patients were seriously obese and perhaps deserved larger doses. A 4-hour dosing interval was the defined desidera- tum. This and slightly compressed schedules are part of surgical lore long heard in Echo Canyon, where "thought leaders" recite to each other cefazolin's half-life and swap campfire tales of tissue levels and computational jujitsu. How certain are the authors that each patient in the alert group had an adequate tissue concentration of cefazolin (ie, above the minimum inhibitory concentration values for expected pathogens) throughout the operation and for at least one Miles-Miles-Burke interval after closure?

Cefazolin-sensitive bacteria lounging around on mediastinal tissues rejoice progressively as cefazolin-rich urine silently drizzles into the Foley catheter bag. I'm disappointed that the authors measured no cefazolin serum levels at different times after $\mathrm{T}=240$ minutes in random samples of patients from each of the groups. It's easy to draw blood from patients connected to the perfusion circuit and even after atrial decannulation there's always a radial arterial line or central venous port within range of the anesthesia team. Wouldn't serum levels have shrewdly showcased the consequences of not using redose alerting?

The authors tacitly used a dichotomy of "adequate" and "bad" prophylaxis. Of 136 cases in the control group, 81 were in the "bad" basket because no cefazolin boost occurred as the magic 240-minute point came and went. We need to see the distribution of case durations for these 81 healthcare customers. Maybe only a few cases are at the extreme end of that distribution ( $T=737$ minutes). This point is worth pondering.

There are no microbiology data. Not all surgical-site infections after cardiac operations are caused by cefazolinsensitive organisms. What about methicillin-resistant Staphylococcus aureus? What about $S$. epidermidis isolates resistant to cefazolin? For patients who had "business as usual" redose patterns in the study, infections caused by organisms resistant to cefazolin darned sure can't be attributed to flawed cefazolin redosing. As well, it is known that isolates from surgical-site infections are not infrequently sensitive to prophylactic antimicrobials that were provably and properly used. ${ }^{5}$ Of course, this bug sensitivity business would have been a non-issue had the authors not decided to discuss the infection rates.

The vulnerability of the article is its inclusion, and subsequent statistical manipulation, of infection rates for the two groups of randomized patients and for a pre-study cardiac case experience. I think the data must have placed a psychological half nelson on the authors just after their discovery of a "significantly" lower infection rate in the study group with ostensibly better prophylaxis when compared with the infection rate for 480 historical "control" operations conducted prior to the clinical trial. Was their data bank visited for a withdrawal of historical group infection information after the $P=.42$ tumbled out from the stochastic contrast of the two study group infection rates? The inference was planted that the lower infection rate in the alerted study arm relative to that found in the historical "controls" meant improved prophylaxis. With due respect, that's a post hoc fallacy until proven otherwise. What data support the implication that cefazolin tissue levels were 
inadequate in historical "control" patients? What bugs were isolated from the 48 infections in that historical experience and how many were resistant to cefazolin? Where is information about obesity, degree of early postoperative glucose control in diabetic patients, distribution of "re-do" sternotomy operations, and other potential risk factor imbalances?

Infection rates weren't needed and should not have been mentioned and here's why. Thousands of cardiac operations are performed each year on planet Earth. For these operations, one either believes that antimicrobial prophylaxis is an indicated process step or one believes that it's elaborate humbug. There is no middle ground. If we believe that the use of prophylaxis is indicated, any randomized, prospective study that seeks to isolate the reduction of a prophylaxis process step error rate stands alone and can speak for itself. ${ }^{6}$ Zanetti et al. were not obligated to test the notion that full-court-press prophylaxis yields a lower infection rate than does half-hearted prophylaxis. Unfortunately, the article's discussion section cryptically conflates outcome and process. Some of its comments may confuse unsophisticated readers not mindful that there is only a probabilistic, not a lock-step, relation between using prophylaxis assiduously and the non-occurrence of surgical-site infections. In the reductionist approach to surgical process improvement, there's a really short list of common ways that prophylaxis can be screwed up: no agent used; worthless agent used; or correct agent used but tissue concentration-time profile not optimal. These are all tightly definable, avoidable process goof-ups and the Zanetti article addressed the third one obliquely.

Alert use and timely cefazolin redosing were indisputably associated, the trial design seems free of shenanigans, and the Harvard folks made a tidy contribution to the literature of "surgical cockpit resource management." That infection rates differed insignificantly between the groups of randomized patients is something we sure didn't need to hear. I worry - as I sit here on my flight to Bermuda-that this tidbit of gossip will hamper marketing of the nifty redose alerting concept.

\section{REFERENCES}

1. Zanetti G, Flanagan HL Jr, Cohn LH, Giardina R, Platt R. Improvement of intraoperative antibiotic prophylaxis in prolonged cardiac surgery by automated alerts in the operating room. Infect Control Hosp Epidemiol 2003;24:13-16.

2. Federal Aviation Regulations, Part 91, Section 91.175.

3. Federal Aviation Administration. Instrument Flying Handbook FAA-H8083-15. Washington, DC: Federal Aviation Administration; 2001.

4. www.chipsplace.com/helpful/Airbus/Airbus320TOC.htm\#TOC.

5. Lee J, Olson M. Wound infection surveillance for 85,260 consecutive operations. Journal of Surgical Outcomes 1999;2:27-42.

6. Lee J. Wound infection surveillance. Infect Dis Clin North Am 1992; 6:643-656. 